\title{
BMJ Open Technology-assisted depression screening tools for patients with cancer: a systematic review protocol
}

\author{
Maria Isabel Lazaro-Escudero (D), Camila Alanna Burgos-Cardona, \\ Karina Acevedo-Fernández, Eida Maria Castro-Figueroa
}

To cite: Lazaro-Escudero Ml, Burgos-Cardona CA, Acevedo-Fernández $\mathrm{K}$, et al. Technology-assisted depression screening tools for patients with cancer: a systematic review protocol. BMJ Open 2021;11:e041878. doi:10.1136/ bmjopen-2020-041878

- Prepublication history and additional materials for this paper is available online. To view these files, please visit the journal online (http://dx.doi. org/10.1136/bmjopen-2020041878).

Received 07 August 2020 Revised 24 December 2020 Accepted 14 January 2021

Check for updates

(c) Author(s) (or their employer(s)) 2021. Re-use permitted under CC BY-NC. No commercial re-use. See rights and permissions. Published by BMJ.

Clinical Psychology Department, Department of Psychiatry, Ponce Health Sciences University, Ponce, Puerto Rico

Correspondence to

Dr Eida Maria Castro-Figueroa; ecastro@psm.edu

\section{ABSTRACT}

Introduction Among patients with cancer, depression is still under-detected. The use of technology-assisted screening tools is rising; however, little is known about the uptake of these devices as depression screening tools among patients with cancer.

Methods and analysis A systematic review will be conducted using the Preferred Reporting Items for Systematic Reviews and Meta-Analysis Protocols (PRISMA-P). The review is registered with PROSPERO and any adjustments to the protocol will be traced. The aims of this systematic review are to (1) identify the most common and feasible depression screening information technology (IT) delivery models among patients with cancer, (2) identify the most common depression screening instrument used in IT devices and (3) describe the published technology-assisted depression screening tools for patients with cancer. PubMed, EBSCOhost and Google Scholar databases will be used. PICO (Patient/

Population, Intervention, Comparison, Outcomes) guidelines will inform the inclusion criteria. Two researchers will independently review titles and abstracts, followed by full article review and data extraction. In the case of a disagreement, a third reviewer will make the final decision. Title/abstract screening will be conducted using a screening tool prepared by the researchers. Articles will be included for review if: (1) the study includes patients with cancer, cancer survivors and/or patients on remission, (2) depression is screened using technology and (3) technology-assisted depression screening effectiveness, efficacy, feasibility and/or acceptance is addressed. The quality of the articles will be assessed using the Methodological Index For Non-Randomised Studies (MINORS, maximum score 24) through independent coding of reviewers.

Ethics and dissemination This research is exempt from ethics approval given that this is a protocol for a systematic review, which uses published data. Findings from this review will be disseminated through peer-reviewed publications and scientific conferences.

Systematic review registration PROSPERO registration number CRD42019121048.

\section{INTRODUCTION}

\section{Rationale}

More than 300million people worldwide suffer from depression. ${ }^{1}$ Depressive spectrum disorders seem to be a common mental health complication of cancer. ${ }^{2}$ A study meta-analysis reported the prevalence of depression symptoms

\section{Strengths and limitations of this study}

This systematic review addresses the use of technology for depression screening in patients with cancer, as well as the process evaluation (feasibility, acceptability, efficacy and/or effectiveness) of information technology delivery models in oncology practice.

- Article screening, data extraction, quality and strength of evidence assessment will be independently conducted by two reviewers to reduce personal bias.

- Studies in languages other than English will not be included or searched, which may contribute to language bias.

in patients with cancer to range from $8 \%$ to $24 \%$, varying due to type of screening instrument, type of cancer and treatment phase. ${ }^{3}$ A large epidemiological multicentre study found that patients with cancer have a fivefold increased chance of experiencing depression in comparison to the general population. ${ }^{4}$ Studies investigating the association between cancer treatments and depression have found a higher prevalence of depressive disorders in patients in active treatment compared with those who are not receiving treatment. ${ }^{2}$ If these numbers are analysed in the context that globally 18.1 million new cases of cancer were identified in $2018,{ }^{15}$ screening depression symptoms among patients with cancer should be a health priority.

The screening of depression is now becoming a significant aspect of cancer care. ${ }^{6}$ For example, the National Comprehensive Cancer Network and the Institute of Medicine have created guidelines and recommendations for routine screening of distress (including depression symptoms) as a quality standard in oncology practice. ${ }^{7}$ It is recommended that those responsible for the care of patients with cancer screen for depression and provide further assessment for patients that screen positive for depression; and the process should identify patients that could 
benefit from additional support. ${ }^{8}$ It has been demonstrated that distress screening improves the management of psychosocial distress in patients with cancer. ${ }^{9}$ Depression symptoms remain under-recognised among patients with cancer and as such they are associated with major effects on patient suffering, mortality and healthcare expenditure. ${ }^{10}$ Despite the existence of evidence-based recommendations to screen and address depression symptoms, depression continues to go under-detected and under-treated. ${ }^{11}{ }^{12}$ One of the main causes of this lack of implementation of depression screening is thought to be limited resources and the proper implementation of this service in most government hospitals. ${ }^{13}$

The use of technology-assisted depression screening tools is beginning to be acknowledged by the US National Institutes of Health $(\mathrm{NIH}) .{ }^{14}$ Compared with the traditional paper-pencil method, electronic devices for symptom assessment have proven to offer diverse benefits such as immediate presentation of results and compilation of aggregate scores, as well as the possibility of tracking symptoms. ${ }^{15}$ The use of screening technology potentiates mental healthcare efficiency by enabling easy access to in-depth follow-up assessment, referral and treatment. ${ }^{16}$ There has been an increased implementation of technology-assisted tools in the cancer care system. ${ }^{17-20}$ The healthcare system has not taken full advantage of the benefits that major advances in computer technology would provide in aiding routine oncology practice. ${ }^{21}$

\section{Objectives}

Little is known about the extent to which IT devices (computers, tablets, mobile phones and so on) are being incorporated into routine depression screening initiatives among patients with cancer. Moreover, we are not aware of any systematic reviews addressing the use of technology for depression screening in patients with cancer. The purposes of this systematic review are to:

1. Identify the most common delivery models of depression screening IT (Internet browser, type of platform, application software and so on) among patients with cancer.

2. Identify the most common instrument used to screen depression in IT devices.

3. Describe the published technology-assisted depression screening tools and explore its feasibility for patients with cancer.

\section{METHODS AND ANALYSIS}

\section{Eligibility criteria}

Study characteristics

\section{Inclusion criteria}

$\mathrm{PICO}^{22}$ (Patient/Population, Intervention, Comparison, Outcomes) guidelines were used to develop inclusion criteria. Articles will be included if they focus on feasibility, acceptability, efficacy or effectiveness of the use of technologyassisted tools to facilitate screening of depression symptoms among patients with cancer seeking medical care (PICOcontext). Articles may include those with randomised control design as long as the comparison group is exposed to other technology-assisted screening tools or the paper and pencil screening format. Also, the protocol includes scientific articles addressing IT-assisted depression screening's feasibility, acceptability and implementation of among patients with cancer. In summary, articles with the following criteria will be included for review:

- Experimental or quasi-experimental research design.

- Randomised and non-randomised control trials.

- Studies involving patients with cancer, subjects who are cancer-free and/or patients on remission.

- Studies using a technology-assisted depression screening tool.

- Studies evaluating the effectiveness, efficacy, feasibility and/or acceptance of the technology-integrated depression screening tool.

- Articles written in English.

\section{Exclusion criteria}

Articles with the following criteria will be excluded for review:

- Studies in which screening technology is used to measure depression prevalence.

- Studies using technology devices to deliver behavioural interventions to treat depression.

- Studies not defining criteria for the measurement of effectiveness, efficacy, feasibility and/or acceptance of the technology-integrated depression screening tool.

- Studies using only qualitative assessments to evaluate effectiveness, efficacy, feasibility and/or acceptance of the technology-integrated depression screening tool.

- Meta-analyses.

\section{Patient and public involvement}

No patients or public will be involved in this study considering it is a systematic review.

\section{Information sources}

\section{Electronic databases}

The following electronic databases will be searched: PubMed, EBSCOhost and Google Scholar.

\section{Additional sources}

Articles will also be obtained from the following sources:

- Reference list of included studies.

- Reference list of an excluded study if it shows direct relevance to fit inclusion criteria.

- Manual searching of articles.

\section{Search strategy}

Search strategy for PubMed is in online supplemental appendix 1 . This search strategy will be adapted to the other databases, reporting further modifications in the review manuscript. Articles published up to December 2020 will be included in this systematic review.

\section{Study selection}

Two main reviewers will independently screen the title and abstract of the articles resulting from keyword search in the three databases. Screening of articles will be made using a 


\begin{tabular}{|c|c|c|c|c|c|c|c|}
\hline $\begin{array}{l}\text { First } \\
\text { author }\end{array}$ & Year & Title & $\begin{array}{l}\text { Patients with cancer, } \\
\text { subjects who are cancer- } \\
\text { free and/or patients on } \\
\text { remission }\end{array}$ & $\begin{array}{l}\text { Depression or } \\
\text { distress screening } \\
\text { using technology }\end{array}$ & $\begin{array}{l}\text { Experimental or quasi- } \\
\text { experimental research } \\
\text { design }\end{array}$ & $\begin{array}{l}\text { IT screening delivery model } \\
\text { effectiveness, efficacy, } \\
\text { feasibility and/or acceptance is } \\
\text { addressed }\end{array}$ & $\begin{array}{l}\text { Articles } \\
\text { written in } \\
\text { English }\end{array}$ \\
\hline
\end{tabular}

IT, information technology.

standardised screening tool, containing the year the study was published, the first author's last name, checkmarks for inclusion criteria and acceptance/rejection of the study. The full text of manuscripts will be obtained if the title and abstract content does not clearly state the inclusion/exclusion criteria. Discrepancies between the two main reviewers will be resolved through discussion and consensus. If there is still discrepancy after discussion, a third reviewer will be added. The third reviewer's selection criteria will also be discussed with the two main reviewers. Duplicates will be removed using the Excel sheet search icon. The criteria for selection or exclusion of all articles will be documented.

\section{Data extraction}

The two review authors will independently extract data from the included studies using an Excel chart that will take into account the inclusion and exclusion criteria (table 1). A practice data extraction section will be performed by reviewers before conducting the data extraction. Any disagreement regarding data extraction will be resolved through discussion and consensus. A third reviewer will resolve any discrepancies.

\section{Data items}

The following information will be extracted:

- Articles' full bibliographical data.

- Model of service delivery type (oncology practice, primary care setting, inpatient setting and so on), screening instrument, type of technology delivery model, primary and secondary outcomes, screening process (with or without confirmed depression diagnosis).

- Sample characteristics and demographics, including sample size, cancer type, nationality and mean age.

- Number of participants, including number of participants per arm in randomised control trials.

- Study methodology, recruitment site and study completion rates.

- Source(s) of research funding and potential conflicts of interest.

- The following parameters will be pooled from each study selected: per cent of 'relapse patients', study design (including exact time points when longitudinal), scale scoring criteria (including cut-off values).

Risk of bias (quality) assessment

The quality of methods in the study will be assessed with the Methodological Index For Non-Randomised Studies (MINORS). ${ }^{23}$ Two independent coders will review all articles included after inclusion/exclusion criteria are applied. Any disagreements will be resolved by discussion that will include a third reviewer. The MINORS tool integrates two levels of assessment, one for quasi-experimental and one for randomised control trials. The following items will be considered for bias assessment:

1. A clearly stated aim.

2. Inclusion of consecutive patients.

3. Prospective collection of data.

4. Endpoints appropriate to the aim of the study.

5. Unbiased assessment of the study endpoint.

6. Follow-up period appropriate to the aim of the study.

7. Loss to follow-up less than $5 \%$.

8. Prospective calculation of the study size.

Additional criteria in the case of comparative study:

1. An adequate control group.

2. Contemporary groups.

3. Baseline equivalence of groups.

4. Adequate statistical analyses.

\section{Data analysis}

Descriptive analysis will be performed to assess frequency and percentage of IT technology tools used for depression screening among patients with cancer, participants' types of cancer and demographic characteristics (sex, age, ethnicity, income). Also, we will calculate the mean age of overall participants. Moreover, we will perform a narrative synthesis of main outcomes based on the extracted data items (table 2).

\section{Grading the strength of evidence}

We will use the GRADE (Grades of Recommendation, Assessment, Development and Evaluation) approach to assess the strength of evidence of the studies reviewed, as recommended by the Cochrane Handbook for Systematic Reviews of Interventions. ${ }^{24}$ This approach evaluates risk of bias, imprecision, inconsistency, indirectness and publication bias. The quality of evidence is rated at all levels: very low, low, moderate and high.

\section{ETHICS AND DISSEMINATION}

Results of this study will be disseminated in scientific conferences and as a peer-reviewed scientific publication. Since the primary data will be extracted from public domain databases, no informed consent of formal ethics committee review is required. 
Table 2 Characteristics of included articles and outcomes

\begin{tabular}{llllllll}
\hline & $\begin{array}{l}\text { Model of } \\
\text { service }\end{array}$ & Year & Number of & Sample characteristics & Screening & Type of & Primary and \\
technology & $\begin{array}{l}\text { Pricle } \\
\text { selivery } \\
\text { published }\end{array}$ & $\begin{array}{l}\text { Narticipants } \\
\text { and demographics }\end{array}$ & instrument & $\begin{array}{l}\text { Study } \\
\text { delivery model }\end{array}$ & outcomes & methodology \\
\hline
\end{tabular}

\section{DISCUSSION}

The National Comprehensive Cancer Network and the Institute of Medicine ${ }^{25}$ have created guidelines and recommendations for routine screening of distress (including depression symptoms) as a quality standard in oncology practice. Despite the existence of such evidence-based recommendations, ${ }^{26}$ depression continues to go under-detected and under-treated. The conclusions drawn by this systematic approach describe progress towards the development of new technology-assisted tools for depression screening in standard oncology practice.

Contributors EMC is the guarantor of this review. MIL-E and EMC conceptualised the systematic review protocol. ML and CB drafted the manuscript, and MIL-E, EMC, $\mathrm{CAB}-\mathrm{C}$ and $\mathrm{KA}-\mathrm{F}$ reviewed the protocol critically.

Funding This work was supported by the National Cancer Institute (2U54CA163071 and 2U54CA163068) and the National Institute of Minority Health and Health Disparities (5G12MD007579-33, 5R25MD007607, R21MD013674 and 2U54MD007579-34).

Competing interests None declared.

Patient consent for publication Not required.

Provenance and peer review Not commissioned; externally peer reviewed.

Supplemental material This content has been supplied by the author(s). It has not been vetted by BMJ Publishing Group Limited (BMJ) and may not have been peer-reviewed. Any opinions or recommendations discussed are solely those of the author(s) and are not endorsed by BMJ. BMJ disclaims all liability and responsibility arising from any reliance placed on the content. Where the content includes any translated material, BMJ does not warrant the accuracy and reliability of the translations (including but not limited to local regulations, clinical guidelines, terminology, drug names and drug dosages), and is not responsible for any error and/or omissions arising from translation and adaptation or otherwise.

Open access This is an open access article distributed in accordance with the Creative Commons Attribution Non Commercial (CC BY-NC 4.0) license, which permits others to distribute, remix, adapt, build upon this work non-commercially, and license their derivative works on different terms, provided the original work is properly cited, appropriate credit is given, any changes made indicated, and the use is non-commercial. See: http://creativecommons.org/licenses/by-nc/4.0/.

\section{ORCID iD}

Maria Isabel Lazaro-Escudero http://orcid.org/0000-0003-3889-9114

\section{REFERENCES}

1 World Health Organization. Depression, 2018. Available: https://www. who.int/en/news-room/fact-sheets/detail/depression

2 Caruso R, Nanni MG, Riba M, et al. Depressive spectrum disorders in cancer: prevalence, risk factors and screening for depression: a critical review. Acta Oncol 2017;56:146-55.

3 Krebber AMH, Buffart LM, Kleijn G, et al. Prevalence of depression in cancer patients: a meta-analysis of diagnostic interviews and selfreport instruments. Psychooncology 2014;23:121-30.

4 Hartung TJ, Brähler E, Faller $\mathrm{H}$, et al. The risk of being depressed is significantly higher in cancer patients than in the general population: prevalence and severity of depressive symptoms across major cancer types. Eur J Cancer 2017;72:46-53.
5 World Health Organization. Cancer, 2018. Available: https://www. who.int/cancer/en/

6 Penedo FJ, Cella D. Responding to the quality imperative to embed mental health care into ambulatory oncology. Cancer 2017;123:382-6.

7 Lowery AE, Holland JC. Screening cancer patients for distress: guidelines for routine implementation. Community Oncol 2011;8:502-5.

8 Smith HR. Depression in cancer patients: pathogenesis, implications and treatment (review). Oncol Lett 2015;9:1509-14.

9 Holland JC, Alici Y. Management of distress in cancer patients. $J$ Support Oncol 2010;8:4-12.

10 Mausbach BT, Irwin SA. Depression and healthcare service utilization in patients with cancer. Psychooncology 2017;26:1133-9.

11 Dauchy S, Dolbeault S, Reich M. Depression in cancer patients. EJC Suppl 2013;11:205-15.

12 Walker J, Hansen $\mathrm{CH}$, Martin P, et al. Prevalence, associations, and adequacy of treatment of major depression in patients with cancer: a cross-sectional analysis of routinely collected clinical data. Lancet Psychiatry 2014;1:343-50.

13 Ingle VK, Pandey I, Singh AR, et al. Screening of patients with chronic medical disorders in the outpatient department for depression using handheld computers as interface and patient health Questionnaire-9 as a tool. Int J Appl Basic Med Res 2017;7:129-33.

14 National Institutes of Health, U.S. Department of Health and Human Services. PA-18-493: using information technology to support systematic screening and treatment of depression in cancer (R01 clinical trial optional). Available: grants.nih.gov/grants/guide/pa-files/ pa-18-493.html

15 Hjermstad MJ, Lie HC, Caraceni A, et al. Computer-based symptom assessment is feasible in patients with advanced cancer: results from an international multicenter study, the EPCRC-CSA. J Pain Symptom Manage 2012;44:639-54.

16 Kingston D, McDonald S, Biringer A, et al. Comparing the feasibility, acceptability, clinical-, and cost-effectiveness of mental health e-screening to paper-based screening on the detection of depression, anxiety, and psychosocial risk in pregnant women: a study protocol of a randomized, parallel-group, superiority trial. Trials 2014;15:3.

17 Aktas A, Hullihen B, Shrotriya S, et al. Connected health: cancer symptom and quality-of-life assessment using a tablet computer: a pilot study. Am J Hosp Palliat Care 2015;32:189-97.

18 Gell NM, Grover KW, Humble M, et al. Efficacy, feasibility, and acceptability of a novel technology-based intervention to support physical activity in cancer survivors. Support Care Cancer 2017;25:1291-300.

19 Quintiliani LM, Mann DM, Puputti M, et al. Pilot and feasibility test of a mobile health-supported behavioral counseling intervention for weight management among breast cancer survivors. JMIR Cancer 2016;2:e4.

20 Stinson JN, Jibb LA, Nguyen C, et al. Construct validity and reliability of a real-time multidimensional smartphone APP to assess pain in children and adolescents with cancer. Pain 2015;156:2607-15.

21 Christ GH, Messner C, Behar LC. Handbook of oncology social work: psychosocial care of the patient with cancer. New York: Oxford University Press, 2015

22 Using PICO or PICo, 2019. Available: https://libguides.murdoch.edu. au/systematic/PICO

23 Dent O. Methodological index for non-randomized studies. ANZ J Surg 2003;73:675-6.

24 Higgins JPT. Cochrane handbook for systematic reviews of interventions. Hoboken, NJ: Wiley-Blackwell, 2020.

25 Holland JC, Andersen B, Breitbart WS, et al. Distress management. $J$ Natl Compr Canc Netw 2013;11:190-209.

26 Mitchell AJ, Vahabzadeh A, Magruder K. Screening for distress and depression in cancer settings: 10 lessons from 40 years of primarycare research. Psychooncology 2011;20:572-84. 